\title{
Effectiveness of biomarkers and serum parameters in determination allergic asthma and detection of its severity
}

\author{
Suhayla H. Shareef ${ }^{*}$, Kawa Amin ${ }^{2}$
}

${ }^{1}$ Department of Microbiology, Immunology, College of Medicine, University of Sulaimania, Sulaimania, Iraq.

${ }^{2}$ Department of Medical Science, Respiratory Medicine, and Allergology. Uppsala University and University

Hospital, Uppsala, Sweden.

*Corresponding author: Suhayla H. Shareef (suhayla.shareef@yahoo.com )

\begin{abstract}
Background and objective: Motivations for exploring appropriate and reliable biomarkers of allergic asthma are early diagnosis, sensitization, asthma endotypes, monitoring of disease and treatment progression. As a result, clinical studies of allergic asthma have focused on measuring biomarkers in order to figure out the characteristics of the population, relevant environmental risk factors, and the effects of various treatments. In this regard, the current study was carried out to examine the effectiveness of different biomarkers in diagnosing allergic asthma and determining its severity.
\end{abstract}

Methods: The current cohort study was conducted at the Allergy and Asthma Center in Sulaimani Hospital on 66 non-smoking patients who had allergic asthma and 61 healthy controls from January 2017 to November 2018. Six ml peripheral venous blood was taken from each subject; $3 \mathrm{ml}$ for assessing total blood count and $3 \mathrm{ml}$ for obtaining the serum. Variables like total and specific serum IgE, levels of serum ECP, Tryptase, serum IL-18, and CD-20, and lung function parameters like FEV1, FVC, and FEC1/FVC were measured. Comparing the unpaired continuous and dichotomous variables was carried out through Mann-Whitney $U$ and Contingency $\chi^{2}$ tests, and spearman's rank coefficient was used for correlation between the parameters. For this purpose, Prism 7.0 Software (Graphpad, La Jolla, California, USA) was used. $\mathrm{p}<0.05$ was considered significant.

Results: The mean age of the patients with allergic asthma and the healthy subject was $39.09 \pm 1.19$ and $39.95 \pm 0.70$ years, respectively. They were significantly different in terms of symptoms $(\mathrm{p}<0.0001)$ and family history $(\mathrm{p}=0.0058)$. Total WBCs, monocyte cells, neutrophile cells, eosinophile cells, and basophile cells are significantly effective and accurate diagnostic tools for allergic asthma $(\mathrm{p}<0.01)$, while lymphocyte cells cannot be a reliable predictor for this disease ( $p>0.05$ ). Asthma, allergy, and inflammation can be diagnosed accurately through serum total IgE, serum tryptase, FEV1, ECP, CD-20, and IL-18. ECP and FEV1 can be used to predict asthma, allergy, and inflammation.

Conclusion: Biomarkers like WBCs, monocyte cells, neutrophile cells, eosinophile cells, and basophile cells are good predictors of allergic asthma, with eosinophile cells being the strongest biomarker. Allergic asthma can also be predicted reliably through IgE, tryptase, FEV1, ECP, CD-20, and IL-18.

Keywords: allergic asthma, serum tryptase, biomarkers, diagnosing, asthma severity and interleukin 


\section{How to cite this article:Shareef SH, Amin K (2020): Effectiveness of biomarkers and serum parameters in determination of allergic asthma and detection of its severity, Ann Trop Med \& Public Health; 23(S18): SP231807. DOI: http://doi.org/10.36295/ASRO.2020.231807}

\section{Introduction}

Asthma is an important public threat that affects over 300 million individuals all over the world ${ }^{(1)}$. It is a non-communicable disease that can result in decreased emotional well-being, limited physical functioning, and poor quality of life ${ }^{(2)}$. Not only the patients but also their families, careers, communities, and the health system can be negatively affected by this disease ${ }^{(3,4)}$.

On other words , asthma is a variable chronic respiratory condition that is characterized by some symptoms like airway hyper-responsiveness (AHR), airway inflammation, airflow limitation, dyspnea, chest tightness, cough, and wheeze ${ }^{(1)}$. Different individuals may develop asthma with different severity from intermittent asthma to severe asthma (SA) which can be a potential threat to the patient's life. Patients with mild-to-moderate asthma can control and treat their condition by self-management education, bronchodilators, and inhaled corticosteroids ${ }^{(5)}$. However, a SA is experienced by $3 \%$ to $10 \%$ of patients, and this form of the disease does not respond to standard or even maximal therapy. Therefore, to prevent a patient with SA from becoming uncontrolled and control his/her disease, there is a need for utmost controller therapy ${ }^{(6)}$. SA leads to social exclusion, limited work capacity or productivity, and reduced physical activity. In addition, increased comorbidity burden has been reported in SA patients ${ }^{(7)}$.

Since the prevalence of asthma is increasing, and there is a serious lack of new therapies, and disease prevention methods are ineffective, there is a dire need for biomarkers that can predict allergic asthma (AA). In this regard, and based on the new clinical guidelines, it is crucial to specify patterns of allergen sensitization and lung function parameters ${ }^{(8)}$. SAcan lead to a broad range of comorbidities which may have a role in disease severity and asthma symptoms, which in turn results in failure to perform an accurate assessment and appropriate treatment. For instance, prevalent comorbidity of asthma is chronic rhinosinusitis which plays a role in disease severity. Asthma is often associated with psychological factors, obesity, and obstructive sleep apnea which make its management difficult ${ }^{(6,9)}$.

Because SA is a complex and heterogeneous disease, advanced approaches are required for its assessment and management ${ }^{(5)}$. As recommended by guidelines for asthma management, initializing therapy and maintaining treatment in a step-wise manner depend on assessing disease severity. If the levels of severity are misclassified, antiinflammatory medications might be underused or overused, which in turn leads to either insufficient asthma control or adverse side-effects as a result of overtreatment ${ }^{(8)}$. Biological therapies have facilitated the recognition of asthma severity level and treatment interventions. However, there are significant barriers to optimal diagnosis, treatment, and management of SAin different health care settings, which are related to organizational systems health, patients, and care providers. In this regard, these barriers need to be tackled in order to facilitate influential evaluation and provide suitable treatment for patients with $\mathrm{SA}^{(10-12)}$.

Biological markers, also called biomarkers, are substances that can be traced in order to assess the functioning of the organs or other health aspects. They are also used to obtain information about the disease 
pathophysiology, the illness course, and/or the response to treatment ${ }^{(13)}$. At their best, biological markers can provide information about the presence or absence of a disease, its severity, its progression, selection of the most effective treatment, and survival possibility of the patient. Biomarkers have long been a significant aspect of medicine, providing useful information, ranging from measuring body temperature to revealing the status of underlying health ${ }^{(14)}$. Measuring pulmonary function provides information about asthma consequence, its severity, and response to treatment; however, they do not always reveal the hidden causes of disease or necessarily the factors playing a role in these processes ${ }^{(15,16)}$.

Given the significance of determining the severity of asthma and the important role that biomarkers can play in this regard, the present study was carried out in order to examine and determine the effectiveness of biomarkers and serum parameters in diagnosing AA and determining its severity.

\section{Materials and Methods}

\section{Study design and setting}

The present investigations were a cohort study that was carried out at the Allergy and Asthma Center in Sulaimania, the Kurdistan region-Iraq from January 2017 to November 2018.

\section{Study population}

The current study was conducted on 66 non-smoking patients who had AA. According to the guideline criteria established by the Global Initiative for Asthma (GINA), a specialist physician diagnosed the patients' AA $^{(17)}$. The patients were selected based on some inclusion criteria, including positivity for specific IgE, increased plasma total IgE levels, a pulmonary function test (PFT), and a minimum of two asthma symptoms (assessed for cough, wheeze, and dyspnea). The exclusion criteria were having other lung-related or medical illnesses, exacerbated asthma, respiratory infection, systemic diseases, chronic pulmonary disorders other than asthma, gastroesophageal reflux disease, cardiac disease, and those treated with systemic corticosteroids within 4 weeks before the study. The study also recruited 61 healthy controls who had similar age and sex to the patients. They were chosen based on the following criteria: (1) no history of respiratory or other diseases that may have interference with the results and (2) baseline forced expiratory volume in 1 second (FEV1) $>80 \%$ predicted and FEV1/forced vital capacity (FVC) ratio $>0.7$.

\section{Blood collection}

Six ml peripheral venous blood was obtained from each subject, of whom $3 \mathrm{ml}$ was separated and put into an EDTA tube for total blood cell count, and the remaining $3 \mathrm{ml}$ was placed in a gel tube to obtain the serum. The tubes have centrifuged at 10,000 rpm for 10 minutes, and the serum was placed into a $1.5 \mathrm{ml}$ Eppendorf tube and stored at $-80^{\circ} \mathrm{C}$ till cytokine level estimation was performed.

Following the manufacturer's protocol, a Human IgE ELISA kit (quantitative solid-phase sandwich enzyme immunoassay technique) provided by Cloud-Clone Corp company (Cloud-Clone Corp, USA) (Catalog number: SEA545Hu) and Inhalation Middle East (IgE) (Euroimmun, UK) (DP 3117-1601E) was used to analyze blood samples and measure total and specific serum IgE. Specific IgE was estimated against Dermatophagoides 
pteronyssinus, Dermatophagoides farinae, Penicillium notatum, Cladosporium herbarum, Aspergillus fumigatus, Candida albicans, Alternaria alternate, Sweet vernal grass, Timothy grass, Cultivated rye, Alder, Birch, Oak, Olive tree, Common ragweed, Mugwort, Dandelion, Cockroach, Cat, and Hamster. The diagnosis of specific IgE of $\geq 0.35$ $\mathrm{kU} / \mathrm{l}$ was utilized as a definition of sensitization to a specific allergen. Sensitization to at least one of the investigated allergens was considered to be IgE sensitization.

Enzyme-linked immune sorbent assay (ELISA) was employed to determine levels of serum concentration ECP ng/mL, Tryptasepg/mL, IL-18 pg/mL, and MS4A1 (CD-20) ng/mL, and the Cloud Clone Corp kit was used to measure tryptase and IL-18 (Cloud-Clone Corp, USA) (Catalog No: SEB070Hu) and (Catalog No: SEA064Hu). For Eosinophil Cationic Protein (ECP), (CUSABIO, USA) (Catalog No: CSB-E11729h) and Membrane Spanning 4 Domains Subfamily A, Member 1 (MS4A1) (CD-20) (MyBioSource, USA) (Catalog No: MBS455171) were employed according to the manufacturer's protocol.

Computerized Spirometer (Jaeger-Toennier, Germany) was used as the PFT to measure FEV1 predicted percent of the patients at their enrollment in the study. Also, a standard spirometry method was used to assess lung function parameters (i.e. FEV1, FVC, and FEV1/FVC).

\section{Statistical analysis}

To compare the unpaired continuous and dichotomous variable Contingency $\chi^{2}$ test and Mann-Whitney U test were used. For various cutoff values, a ROC curve was employed to indicate the features of a diagnostic test by graphing the true-positive rate (sensitivity) on the vertical axis and the false-positive rate (1-specificity) on the horizontal axis. Correlations among the continuous variables were defined using Spearman's rank correlation analysis. The contributions of continuous variables were identified using linear regression analysis. Also, the receiver operating characteristic (ROC) analysis was performed. To determine the cutoff points, the likelihood ratio statistic was used, which assess the value of the test for increasing certainty about a positive diagnosis. To calculate the likelihood ratio statistic, [sensitivity/(1-specificity)] was used ${ }^{(18)}$. All statistical analyses were performed through Prism 7.0 Software (Graphpad, La Jolla, California, USA). The statistically significant level was set as $\mathrm{P}<0.05$.

\section{Ethical considerations}

All of the subjects gave informed consent prior to their participation in the study. As well as, the Ethics Scientific Committee of the College of Medicine, Sulaimania University, Iraq approved the study protocol.

\section{Results}

According to the results of the study, AA patients and the healthy controls (HC), mean age was 39.09 and 39.95 years, respectively, and there was no significant difference between them in this regard $(\mathrm{p}=0.54)$. Most of the patients were females in both groups with $63.64 \%$ and $65.57 \%$ in the AA and HC, respectively, and they were not significantly different in this regard $(\mathrm{p}=0.81)$. The two groups were significantly different with respect to their symptoms ( $\mathrm{p}<0.0001)$ and family history $(\mathrm{p}=0.0058)$. The duration of asthma was found to be $9.0 \pm 1.113$ years with a range from 1 to 34 years (See Table 1).

Table 1. Demographic characteristics of the study subjects

\section{Annals of Tropical Medicine \& Public Health http://doi.org/10.36295/ASRO.2020.231807}




\begin{tabular}{|c|c|c|c|}
\hline $\mathrm{F}_{\text {Variables }}$ Groups & $\begin{array}{l}\text { AA patients } \\
\qquad(\mathrm{n}=66)\end{array}$ & $\begin{array}{l}\text { Healthy control } \\
\qquad(\mathrm{n}=61)\end{array}$ & $p$-value \\
\hline Age (yr; Mean \pm SE) & $39.09 \pm 1.19$ & $39.95 \pm 0.70$ & $\begin{array}{c}0.543 \\
n s\end{array}$ \\
\hline Gender $(\mathrm{N}, \%)$ & & & \\
\hline Male & $24(36.36)$ & $21(34.43)$ & 0.819 \\
\hline $\begin{array}{c}\text { Symptoms (Mean } \pm \text { SE) } \\
\text { (cough, wheezes, dyspnea) } \\
1 \text { symptom }\end{array}$ & $\begin{array}{c}2.591 \pm 0.080 \\
6(9.09)\end{array}$ & $0.131 \pm 0.043$ & $<0.0001 * * * *$ \\
\hline \begin{tabular}{|} 
Family History (Mean \pm SE) \\
Non Relative \\
$1^{\text {st }}$ Relative \\
$2^{\text {nd }}$ Relative \\
$3^{\text {rd }}$ Relative
\end{tabular} & $\begin{array}{c}0.681 \pm 0.120 \\
39(59.09) \\
15(22.72) \\
6(9.09) \\
6(9.09)\end{array}$ & $\begin{array}{c}0.245 \pm 0.095 \\
54(88.52) \\
2(3.28) \\
2(3.28) \\
3(4.92)\end{array}$ & $0.0058 * *$ \\
\hline $\begin{array}{l}\text { Duration of Asthma } \\
\qquad(\mathrm{yr} ; \text { Mean } \pm \mathrm{SE})\end{array}$ & $\begin{array}{c}\text { Range (1-34) } \\
9.0 \pm 1.113\end{array}$ & NA & - \\
\hline
\end{tabular}

A ROC curve was constructed for the candidate whole leukocyte blood cells to AA and HC. According to the results obtained for sensitivity versus specificity on ROC curve plots and the area under the curve (AUC), it was seen that total WBCs, monocyte cells, neutrophile cells, eosinophile cells, and basophile cells are effective and accurate diagnostic tools for AA. Eosinophile cells had the highest AUC value for predicting AA. However, lymphocyte cells with the lowest AUC value cannot be a reliable predictor for AA (See Figure 1). 

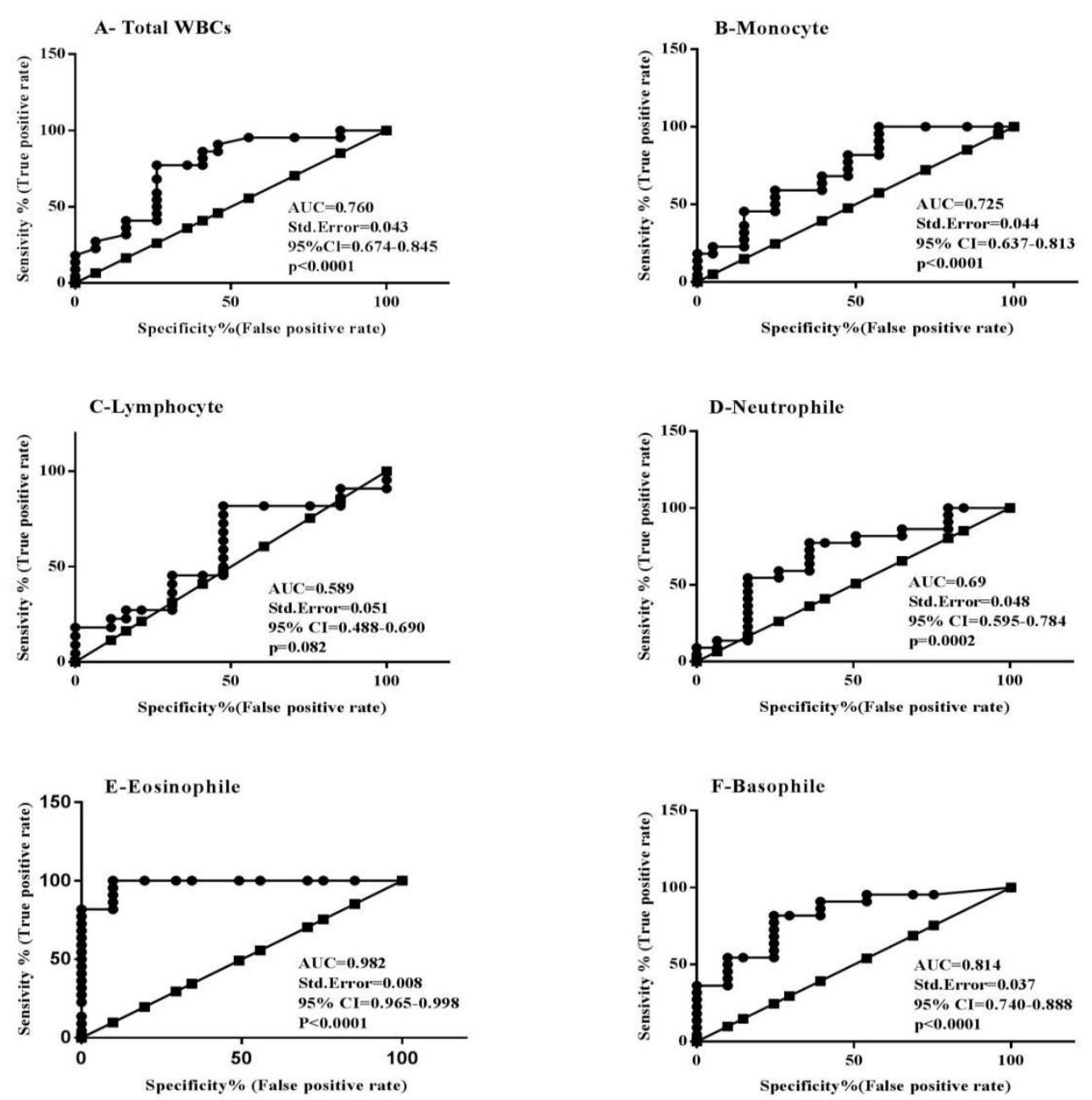

Fig. 1. ROC curves of whole leukocyte blood cells for predicting allergic asthma. A) Total WBCs, B) Monocyte cells, C) Lymphocyte cells, D) Neutrophile cells, E) Eosinophile cells, and F) Basophile cells

According to the results obtained from sensitivity versus specificity on ROC curve plots and the AUC, serum total IgE level, serum tryptase level, and FEV1 are the significantly accurate parameter for predicting AA, serum ECP level and serum CD-20 level for predicting allergy, and serum Interleukin-18 (IL-18) level for predicting inflammation. FEV1 with the highest AUC value is the strongest tool for predicting asthma (See Figure 2). 

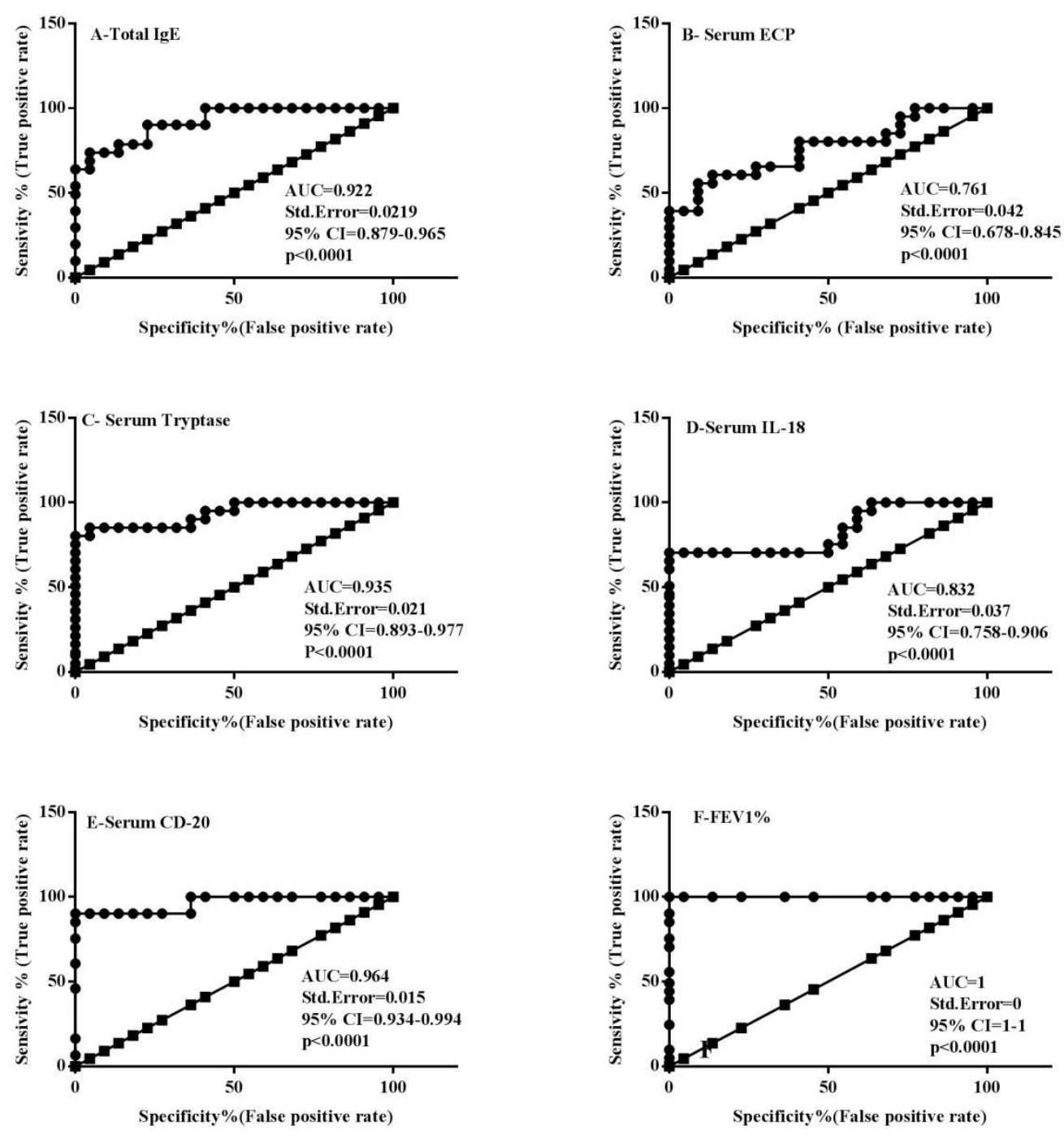

Fig. 2. ROC curves of parameters for predicting allergic asthma. A) Serum total IgE level for predicting allergic asthma, B) Serum ECP level for predicting allergy, C) Serum tryptase level for predicting asthma, D) Serum IL-18 level for predicting inflammatory, E) Serum CD-20 level for predicting allergy, and F) FEV1 for predicting asthma

The correlation between ECP and serum biomarkers in AA patients has done by utilizing linear regression. The findings indicated that ECP has a significant positive association with IgE, tryptase, IL-18, and CD20. Therefore, ECP can be a reliable predictor for asthma, allergy, and inflammation (See Figure 3). 

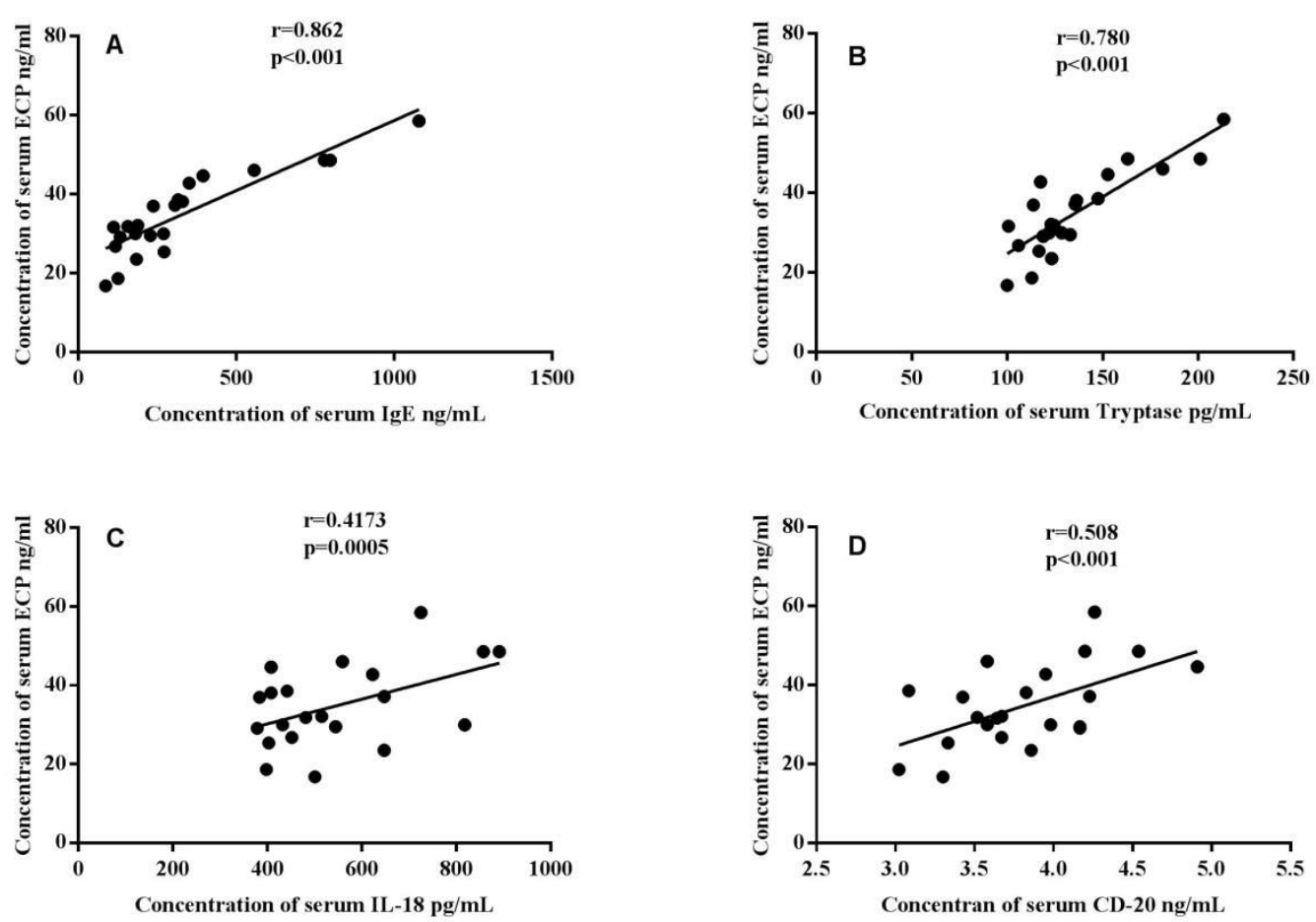

Fig. 3. Linear regression for correlation between ECP and serum-biomarkers. A) Association between ECP and IgE, B) Association between ECP and tryptase, C) Association between ECP and IL-18, D) Association between ECP and CD-20

Correlation between the studied biomarkers was examined using linear regression. The results revealed that serum IgE in patients with AA had a significant positive correlation with serum tryptase, serum IL-18, and serum CD-20. Serum tryptase had a significant positive correlation with serum IL-18 and serum CD-20. Serum IL-18 and serum CD-20 had a significant positive correlation together (See Figure 4). 

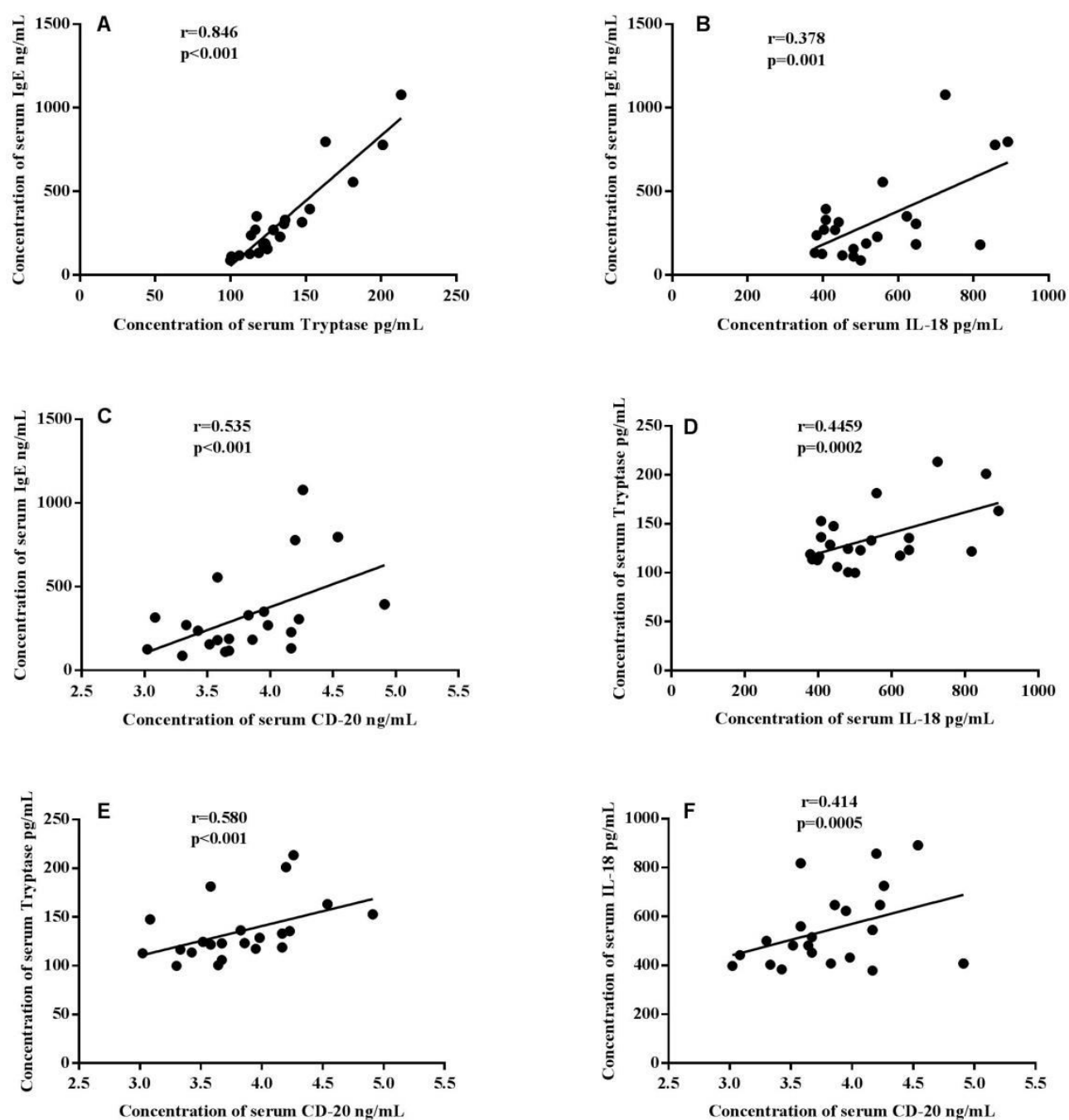

Fig. 4. Linear regression for correlation between serum-biomarkers. A) Association between total IgE and tryptase, B) Association between total IgE and IL-18, C) Association between total IgE and CD-20, D) Association between Tryptase and IL-18, E) Association between Tryptase and CD-20, F) Association between IL-18 and CD-20

The correlation between FEV1 and serum biomarkers was examined using linear regression. The results revealed that FEV1 had a significant negative association with ECP, IgE, tryptase, IL-18, and CD-20 in patients with AA. Therefore, asthma, allergy, and inflammation in patients with AA can be diagnosed through FEV1 (See Figure 5). 

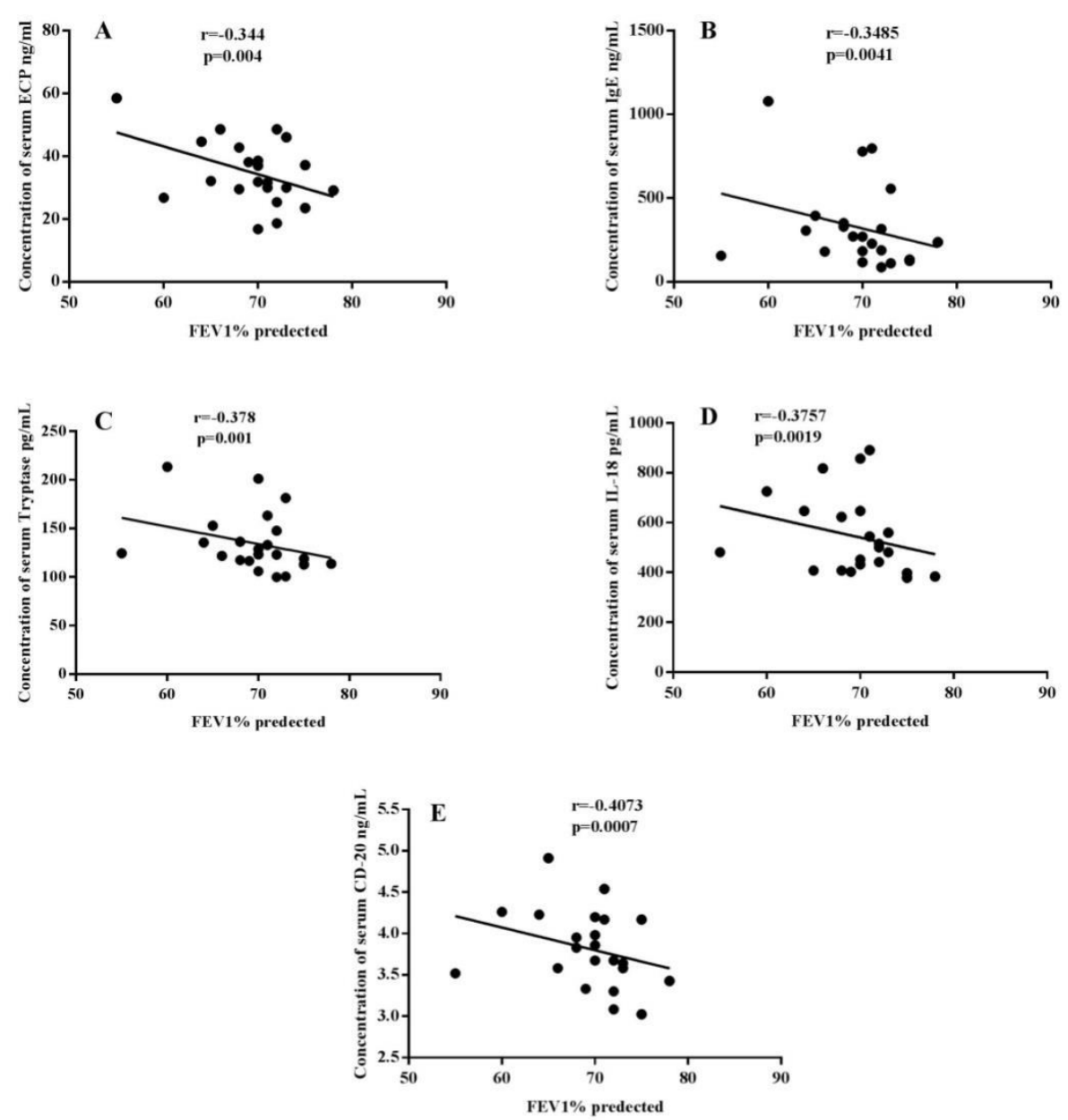

Fig. 5. Linear regression for correlation between FEV1 and serum biomarkers. A) Association between FEV1 and serum ECP, B) Association between FEV1 and total serum IgE, C) Association between FEV1 and serum Tryptase,

D) Association between FEV1 and serum IL-18, E) Association between FEV1 and serum CD-20

\section{Discussion}

The current therapeutic approach for asthma is effective in a number of patients; however, patients with uncontrolled SA need to be provided with a wider range of treatment options. Some studies have proposed utilizing specific phenotype characteristics and biomarkers to manage SA which is difficult to treat ${ }^{(19)}$. According to the data obtained in the present study, it was revealed that cough, wheezes, and dyspnea are the most frequent symptoms associated with AA. This finding is in line with those reported by Kushnir et al (2015) and Mehta (2018)they showed that asthma might be associated with symptoms such as a heavy and vague feeling of chest tightness. They also stated that AA can be associated with symptoms of conjunctivitis and rhinitis. Other symptoms pointed by them are dyspnea, chest tightness, wheezing, and coughing ${ }^{(20,21)}$. Hizawa et al (2015) pointed out that genes play a remarkable role in the transfer of epithelial damage to airway inflammation activation and the adaptive immune system, and that family and heritability play a strong role in the development of $60 \%$ of the cases ${ }^{(22)}$. In line with this, the results of the present study showed that there was a significant difference between the two groups in terms of their symptoms and family history. 
As data obtained in the present study indicated, the WBCs, monocyte cells, neutrophile cells, eosinophile cells, and basophile cells are effective and accurate diagnostic tools for AA. In addition, eosinophile cells play an impressive role in predicting AA. In line with this finding, studies have shown that circulating components of the immune system including mononuclear cells (NK cells, dendritic cells, monocytes, and lymphocytes) and Polymorphonuclear cells or granulocytes (basophils, eosinophils, and neutrophils) can be significantly used for accurate diagnosis of $\mathrm{AA}^{(23,24)}$.

Some features that are specific to asthma-like allergic airway inflammation, heightened immune response, AHR, and airway remodeling are caused by the eosinophil cells. As a specific feature of AA, eosinophilic inflammation has been reported to be more common in childhood than adulthood onset ${ }^{(25,26)}$. If this phase becomes chronic, the lung will be remodeled. Moreover, the deposition of the extracellular matrix leads to damage to airway epithelium. Moreover, in individuals with asthma, alveolar collapse is prevented as a result of the secretion of phospholipid surfactants by epithelial cells at the interface of the airway and alveoli ${ }^{(27)}$. The secretion of $\operatorname{IgE}$ antibodies is one of the significant characteristics of the humoral response. Inflammatory mediators are secreted as a result of the binding of the allergen to the total IgE level on the surface of mastocytes and basophils. Mastocytes include granule which acts on neurons to cause bronchoconstriction and goblet cells to mucus production ${ }^{(28,29)}$.

The result of the present study also demonstrated that serum total IgE level and serum tryptase level are the most accurate parameters for predicting AA. Similar to this finding, De Schryver et al (2016) reported that the most severe phenotype of IgE-mediated hypersensitivity is anaphylaxis, and most cases can be diagnosed by the rise in serum tryptase which is a helpful biomarker ${ }^{(30)}$. It has also been shown that there is a moderate correlation between serum periostin and sputum eosinophilia ${ }^{(31)}$. Research has indicated that ECP can predict sputum eosinophilia more effectively than serum IgE; however, there is a poor correlation between C-reactive protein and sputum neutrophilia ${ }^{(32)}$. These results are in agreement and could be considered as a proof to the findings of the current study that showed ECP can be a reliable predictor for asthma, allergy, and inflammation.

The results of the current study revealed that serum IgE in patients with AA had a significant positive correlation with serum tryptase, serum IL-18, and serum CD-20. On the contrary, in their study, Shin et al (2005) examined how IL18 polymorphisms exert genetic effects on total serum IgE and specific IgE levels and reported that they are not significantly correlated in asthmatic patients ${ }^{(33)}$. Moreover, as demonstrated by Shaaban et al (2014), IL18 would lead to a dramatic rise in airway mucus, eosinophil application in the airways, IgE levels, and Th2 cytokine production. They also examined IL18 expression functions and serum levels in allergic diseases and concluded that patients with SA and AA had increased serum IL18 levels ${ }^{(34)}$.In their study, Prathap et al (2016) assessed the association effectiveness of the release of basophil histamine as an asthma marker. For this purpose, they measured the responsiveness of the airway to histamine via provocative concentration needed to reduce FEV 1 by $20 \%$ (PC20). However, the results of their study showed a significant inverse relationship between PC20 and histamine and three concentrations of anti- $\operatorname{IgE} \mathrm{E}^{(35)}$.

As demonstrated by the results of the current study, inflammation, allergy, and asthma in AA patients could be diagnosed with the help of baseline forced expiratory volume 1. However, there was a considerable negative association among FEV 1 and ECP, IgE, tryptase, IL-18, and CD-20 in patients with AA. In this regard, Chanez et al (2010) showed that any response in the late phase like a decrease in FEV1 of 15\% or more about 6 hours after 
challenge might be an appropriate predictor for airway eosinophilia which is in line with the results of the present study ${ }^{(36)}$.

\section{Conclusion}

AA can be diagnosed accurately through WBCs, monocyte cells, neutrophile cells, eosinophile cells, and basophile cells, while lymphocyte cells cannot be a reliable predictor for allergic asthma. Eosinophile cells had the highest AUC value for predicting AA. Moreover, IgE level, serum tryptase level, and FEV1 are the accurate parameters for predicting AA, ECP level and CD-20 level for predicting allergy, and IL-18 level for predicting inflammation. FEV1 with the highest AUC value is the strongest tool for predicting asthma. Significant positive correlations were found between IgE serum tryptase, serum IL-18, and serum CD-20, between serum tryptase and serum IL-18 and serum CD-20, and between serum IL-18 and serum CD-20. FEV1 has a significant negative relationship with ECP, IgE, IL-18, and CD-20.

\section{References}

1. Scherzer R, Grayson MH. Heterogeneity and the origins of asthma. Annals of Allergy, Asthma \& Immunology. 2018;121(4):400-5.

2. McDonald VM, Hiles SA, Jones KA, Clark VL, Yorke J. Health-related quality of life burden in severe asthma. Medical Journal of Australia. 2018;209(S2):S28-S33.

3. Cordova-Rivera L, Gibson PG, Gardiner PA, Powell H, McDonald VM. Physical activity and exercise capacity in severe asthma: key clinical associations. The Journal of Allergy and Clinical Immunology: In Practice. 2018;6(3):814-22.

4. Foster JM, McDonald VM, Guo M, Reddel HK. "I have lost in every facet of my life": the hidden burden of severe asthma. European Respiratory Journal. 2017;50(3):1700765.

5. Reddel HK, Bateman ED, Becker A, Boulet L-P, Cruz AA, Drazen JM, et al. A summary of the new GINA strategy: a roadmap to asthma control. European Respiratory Journal. 2015;46(3):622-39.

6. Chung KF, Wenzel SE, Brozek JL. Comment on: International ERS/ATS guidelines on definition, evaluation and treatment of severe asthma. European Respiratory Journal. 2014;44(1):267-8.

7. Hiles SA, Harvey ES, McDonald VM, Peters M, Bardin P, Reynolds PN, et al. Working while un well: workplace impairment in people with severe asthma. Clinical \& Experimental Allergy. 2018;48(6):650-62.

8. Mahmood MA, Rashid BM, Amin KA, Tofiq DM, Nore BF. Correlation between serum tryptase level and disease severity in asthmatic patients in the sulaimani governorate. International Journal of Medical Research \& Health Sciences. 2016;5(6):34-41.

9. Wark PA, Hew M, Maltby S, McDonald VM, Gibson PG. Diagnosis and investigation in the severe asthma clinic. Expert review of respiratory medicine. 2016;10(5):491-503.

10. McDonald VM, Maltby S, Reddel HK, King GG, Wark PA, Smith L, et al. Severe asthma: current management, targeted therapies and future directions—a roundtable report. Respirology. 2017;22(1):53-60.

11. Chung LP, Johnson P, Summers Q. Models of care for severe asthma: the role of primary care. Medical Journal of Australia. 2018;209(S2):S34-S40.

12. Chung LP, Hew M, Bardin P, McDonald VM, Upham JW. Managing patients with severe asthma in Australia: Current challenges with the existing models of care. Internal medicine journal. 2018;48(12):1536-41. 
13. Lapraz J-C, Hedayat KM, Pauly P. Endobiogeny: a global approach to systems biology (Part 2 of 2). Global advances in health and medicine. 2013;2(2):32-44.

14. Strimbu K, Tavel JA. What are biomarkers? Current Opinion in HIV and AIDS. 2010;5(6):463.

15. Lyons TJ, Basu A. Biomarkers in diabetes: hemoglobin A1c, vascular and tissue markers. Translational Research. 2012;159(4):303-12.

16. Brumme Z, Wang B, Nair K, Brumme C, de Pierres C, Reddy S, et al. Impact of select immunologic and virologic biomarkers on CD4 cell count decrease in patients with chronic HIV-1 subtype C infection: results from Sinikithemba Cohort, Durban, South Africa. Clinical infectious diseases. 2009;49(6):956-64.

17. Bateman ED, Hurd S, Barnes P, Bousquet J, Drazen J, FitzGerald M, et al. Global strategy for asthma management and prevention: GINA executive summary. European Respiratory Journal. 2008;31(1):143-78.

18. Tilbury JB, Van Eetvelt W, Garibaldi JM, Curnsw J, Ifeachor EC. Receiver operating characteristic analysis for intelligent medical systems-a new approach for finding confidence intervals. IEEE Transactions on Biomedical Engineering. 2000;47(7):952-63.

19. Zervas E, Samitas K, Papaioannou AI, Bakakos P, Loukides S, Gaga M. An algorithmic approach for the treatment of severe uncontrolled asthma. ERJ open research. 2018;4(1):00125-2017.

20. Kushnir NM, Kaliner MA, FAAAAI M, Scarupa MD, Chase C. In-Depth Review of Allergic Rhinitis. 2015 .

21. Mehta R. Allergy and Asthma: Allergic Rhinitis and Allergic Conjunctivitis. FP essentials. 2018;472:11-5.

22. Hizawa N. Genetics of Asthma. Journal of General and Family Medicine. 2015;16(4):252-9.

23. Hudon Thibeault A-A, Laprise C. Cell-Specific DNA Methylation Signatures in Asthma. Genes. 2019;10(11):932.

24. Alculumbre S, Pattarini L. Purification of human dendritic cell subsets from peripheral blood. Dendritic Cell Protocols: Springer; 2016. p. 153-67.

25. Possa SS, Leick EA, Prado CM, Martins MA, Tibério IFLC. Eosinophilic inflammation in allergic asthma. Frontiers in pharmacology. 2013;4:46.

26. de Nijs SB, Venekamp LN, Bel EH. Adult-onset asthma: is it really different? European Respiratory Review. 2013;22(127):44-52.

27. Winkler C, Hohlfeld JM. Surfactant and allergic airway inflammation. Swiss medical weekly. 2013;143(3132).

28. Walsh ER, Stokes K, August A. The role of eosinophils in allergic airway inflammation. Discovery medicine. 2010;9(47):357-62.

29. Sindher SB, Long A, Acharya S, Sampath V, Nadeau KC. The use of biomarkers to predict aero-allergen and food immunotherapy responses. Clinical reviews in allergy \& immunology. 2018;55(2):190-204.

30. De Schryver S, Halbrich M, Clarke A, La Vieille S, Eisman H, Alizadehfar R, et al. Tryptase levels in children presenting with anaphylaxis: temporal trends and associated factors. Journal of Allergy and Clinical Immunology. 2016;137(4):1138-42.

31. Wagener AH, de Nijs SB, Lutter R, Sousa AR, Weersink EJ, Bel EH, et al. External validation of blood eosinophils, FENO and serum periostin as surrogates for sputum eosinophils in asthma. Thorax. 2015;70(2):115-20. 
32. Howrylak JA, Moll M, Weiss ST, Raby BA, Wu W, Xing EP. Gene expression profiling of asthma phenotypes demonstrates molecular signatures of atopy and asthma control. Journal of Allergy and Clinical Immunology. 2016;137(5):1390-7. e6.

33. Shin H, Kim L, Park B, Choi Y, Park HS, Hong SJ, et al. Association of interleukin 18 (IL18) polymorphisms with specific IgE levels to mite allergens among asthmatic patients. Allergy. 2005;60(7):900-6.

34. Shaaban HH, Mohy AM, Abdel-Razek A-RA, Wahab AA. Interleukin-18-607C/A Gene Polymorphism in Egyptian Asthmatic Children. Molecular diagnosis \& therapy. 2014;18(4):427-34.

35. Pillai P, Chan Y-C, Wu S-Y, Ohm-Laursen L, Thomas C, Durham SR, et al. Omalizumab reduces bronchial mucosal IgE and improves lung function in non-atopic asthma. European Respiratory Journal. 2016;48(6):1593-601. 36. Chanez P, Contin-Bordes C, Garcia G, Verkindre C, Didier A, De Blay F, et al. Omalizumab-induced decrease of FceRI expression in patients with severe allergic asthma. Respiratory medicine. 2010;104(11):1608-17. 